\title{
Tunneling Energy Effects in Photoinduced Charge and Energy Transfer
}

\author{
Oliver S. Wenger*
}

\begin{abstract}
Driving force effects on electron transfer rates in molecular donor-bridge-acceptor systems have been investigated extensively in the past. Significantly less well explored are tunneling energy effects in long-range charge transfer. These effects have to do with the energy differences between the donor/acceptor energy levels and those of the bridging molecules that are in between the two reactants. This contribution summarizes some recent advances in this research field.
\end{abstract}

Keywords: Charge separation · Electron transfer · Electronic coupling · Superexchange · Tunneling

If one is to prepare artificial molecular systems that emulate natural photosynthesis, thorough comprehension of all factors governing electron transfer (ET) rates is essential. According to Marcus theory there are three parameters that determine these reaction rates: ${ }^{[1]}$

i) the free-energy for the ET reaction $\left(\Delta \mathrm{G}^{0}\right)$

ii) a parameter $(\lambda)$ related to inner shell and solvent reorganization accompanying the charge redistribution, and

iii) the electronic coupling $\left(\mathrm{H}_{\mathrm{DA}}\right)$ between the donor and the acceptor.

One of the key predictions of this theory, tested successfully in various experiments on suitable donor-bridge-acceptor (D-b-A) molecules, ${ }^{[2,3]}$ is a Gaussian freeenergy dependence of ET rates. Based on these theoretical and experimental studies it appears reasonable to conclude that in order to obtain long-lived charge-separated states that are needed for light-to-chem-

${ }^{*}$ Correspondence: O. S. Wenger

Département de Chimie Minérale, Analytique et Appliquée

Université de Genève

30, quai Ernest-Ansermet

$\mathrm{CH}-1211$ Genève 4

Tel.: +4122 3796051

Fax: +41223796069

E-Mail: oliver.wenger@unige.ch ical-energy conversion, it is desirable to take advantage of the Gaussian free-energy dependence of ET rates and the interplay between $\Delta \mathrm{G}^{0}$ and $\lambda$. More specifically, a light-induced charge-separation (CS) reaction, leading to a state with a hole on the donor and an electron on the acceptor, should be driving force optimized at $\Delta \mathrm{G}_{\mathrm{CS}}{ }^{0}$ $=\lambda$ whereas the reverse process, charge recombination $(\mathrm{CR})$, should fall into the so-called inverted region where $\Delta \mathrm{G}_{\mathrm{CR}}{ }^{0}>\lambda$. In this scenario, $\mathrm{CS}$ rates can exceed $\mathrm{CR}$ rates by several orders of magnitude even in relatively simple artificial D-b-A molecules. ${ }^{[3]}$ While numerous experimental studies on molecular D-b-A systems have focused on this design strategy, the question arises whether discrimination between the rates of desired ET reactions (CS) and undesired energy-wasting ETs (CR) could be amplified by other means. Theoretical work from the 1960s as well as recent experimental studies suggest that this is so. ${ }^{[4]}$ Indeed, so-called differential electronic coupling may offer an additional important way to favor energy-storing over energy-wasting ETs: If electronic donor-acceptor coupling $\mathrm{H}_{\mathrm{DA}}$ is stronger for photoinduced CS than for thermal $\mathrm{CR}$, the rate of the former process will exceed the rate of the latter. Since electron tunneling rates are proportional to the square of the electronic coupling, ${ }^{[1]}$ even quite moderate differences in $\mathrm{H}_{\mathrm{DA}}$ can be expected to lead to a marked effect.

According to superexchange theory for long-range electron tunneling, the electronic coupling $\mathrm{H}_{\mathrm{DA}}$, reflecting the strength of the interaction between reactants and products at the transition state configuration, depends on four parameters (Fig. 1): ${ }^{[4]}$ the electronic coupling strengths between the two redox centers and the bridge $\left(\mathrm{h}_{\mathrm{Db}}, \mathrm{h}_{\mathrm{bA}}\right)$, the coupling between adjacent (and in this model identical) bridging units $\left(\mathrm{h}_{\mathrm{bb}}\right)$, and the so-called tunneling energy gap $\Delta \varepsilon$ :

$$
H_{D A}=\frac{h_{D b}}{\Delta \varepsilon} \cdot\left(\frac{h_{b b}}{\Delta \varepsilon}\right)^{n-1} \cdot h_{b A}
$$

This latter parameter is defined as the energy required to remove an electron from the donor, or hole from the acceptor, and place it on the bridge. It can therefore be regarded as a measure of the barrier height associated with the electron (or hole) tunneling process, but it is important to note that $\Delta \varepsilon$ is the vertical energy gap at the transition state configuration (Fig. 1), i.e. a quantity that is not readily extracted from experiment. This simple model predicts that superexchange ET rates will display an exponential distance dependence. The decay constant, $\beta$, depends on $h_{b b}, \Delta \varepsilon$ and the length of the repeating bridge unit $(\alpha)$ according to

$$
\beta=\frac{2}{\alpha} \cdot \ln \left(\frac{\Delta \varepsilon}{h_{b b}}\right)
$$

The $\beta$ value is in fact the experimentally accessible parameter that is most sensitive to changes in $\Delta \varepsilon$; therefore it is the distance dependence of ET rates that must be evaluated to obtain information on the magnitude of $\Delta \varepsilon$. Indeed, several recent experimental studies tried to observe tunneling energy effects by investigating the distance dependences of electron and hole tunneling in arti- 


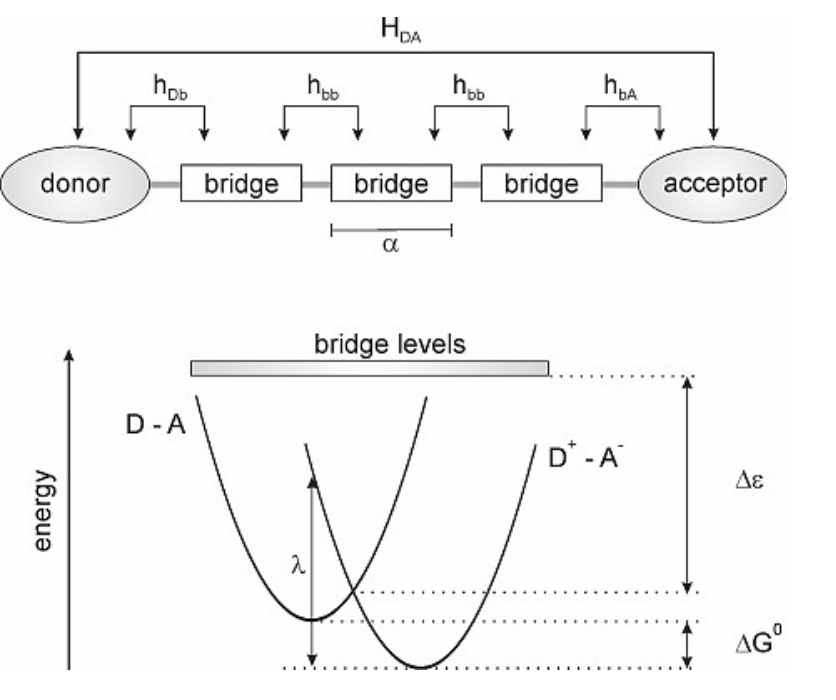

Fig. 1. Graphical illustration of the individual parameters of the superexchange model (Eqns (1) and (2)) for charge transfer ${ }^{[4]}$

ficial D-b-A systems that were specifically designed for this purpose. This means first and foremost that these investigations focused on rigid bridging molecules that have more or less length-independent energy levels. This permits investigation of fixeddistance ET, and the transition from single step to multistep tunneling ('hopping') upon bridge lengthening is prevented. ${ }^{[5]}$ Beratan and coworkers focused on photoinduced charge tunneling in DNA hairpins. ${ }^{[6,7]}$ Specifically, they investigated ET from guanine $(G)$ and deazaguanine $(Z)$ donors to photoexcited stilbene-dicarboxamide (SA) and phenanthrene-dicarboxamide (PA) acceptors through intervening adenine (A) thymine (T) base pairs (Fig. 2, left). Simple energetic considerations led to the conclusions that in these systems i) the charge transfer must occur via a hole tunneling mechanism, and

ii) that this mechanism primarily invokes the adenine (and not the thymine) bridging units.

The remarkable finding of this study is that the distance dependence for charge transfer in the SA-adenine $-\mathrm{n}$ system is significantly weaker than for the PA-adenine ${ }_{n}-$ $\mathrm{Z}$ dyads (Fig. 2, upper right); the $\beta$ distance decay parameters are $0.71 \AA^{-1}$ for the former and $1.1 \AA^{-1}$ for the latter. ${ }^{[6]}$ The driving force $\Delta \mathrm{G}^{0}$ for charge transfer is identical in both systems; the key difference between them is the tunneling energy gap $\Delta \varepsilon$ (Fig. 2, lower right): Based on the redox potentials of the relevant hole donors (SA, PA) and bridging units (adenine) and based on the energies of the singlet excited states of the two hole donors (SA, PA), injection of a hole from photoexcited SA to adenine is calculated to be endergonic by $0.25 \mathrm{eV}$ whereas hole injection from photoexcited PA to adenine is estimated to be energetically uphill by 0.50 $\mathrm{eV}$. While these values do not correspond directly to those of $\Delta \varepsilon$, see above, it appears obvious from these calculations that the experimentally observed differences in longdistance charge transfer efficiencies are due to a tunneling-energy effect - particularly in view of the fact that the driving force for charge transfer is the same in the two types of investigated D-b-A dyads.

Albinsson and coworkers along with the groups of Mårtensson and Anderson performed a series of systematic experimental and theoretical investigations of bridgemediated electronic couplings in porphyrin containing D-b-A molecules. In a recent theoretical study, these researchers calculated the distance dependences of the electronic D-A coupling for triplet energy transfer through oligo(phenyleneethynylene) (OPE) bridges for a variety of different D-A pairs. ${ }^{8]}$ The key finding was a remarkably strong dependence of long-distance energy transfer rates on the energy differences between the triplet levels of the donor and those of the OPE bridge: For small energy gaps ( $0.1 \mathrm{eV}$ ) such as the case for an acridine donor weak distance dependences were found $\left(\beta \sim 0.1 \AA^{-1}\right)$, whereas large energy gaps $(\sim$ $1 \mathrm{eV})$ such as the case for a pentacene donor lead to distance decay constants on the order of $\sim 0.5 \AA^{-1}$ (Fig. 3). Even the change from a zinc(II) porphyrin to a free base porphyrin donor is calculated to affect drastically the long distance triplet energy transfer rates across OPE bridges; the predicted $\beta$-values are $0.19 \AA^{-1}$ for the former and $0.32 \AA^{-1}$ for the latter. At a $25 \AA$ donor-acceptor distance this translates into a two order of magnitude difference in energy transfer rates. In their
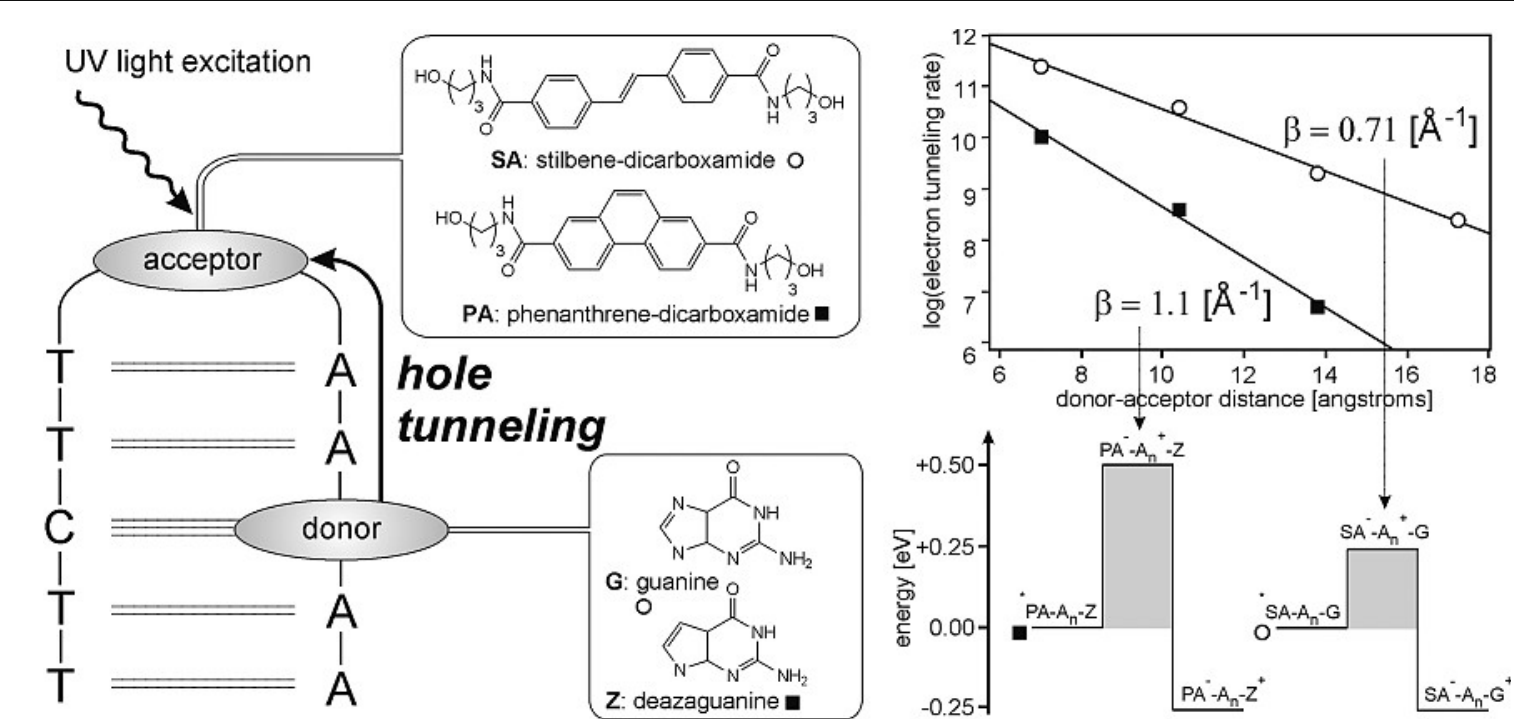

Fig. 2. Left: Structure of DNA hairpins with donors and acceptors for photoinduced charge tunneling investigations. ${ }^{[6,7]}$ Upper right: Experimentally determined distance dependence of charge transfer with two different donor-acceptor pairs through the same DNA hairpin. Lower right: Energetics of bridge mediated charge transfer between the two different donor-acceptor pairs. 


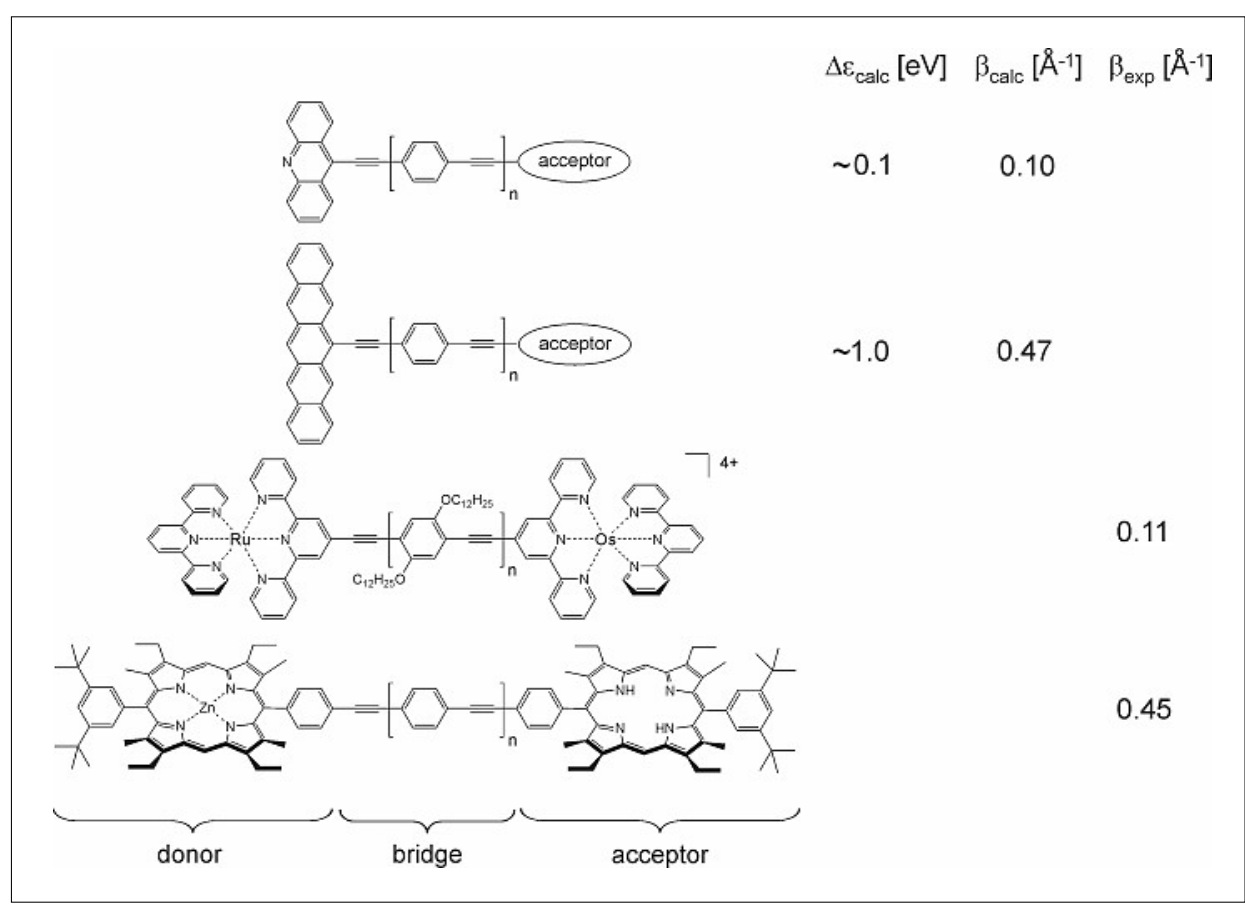

Fig. 3. Calculated and experimental distance decay parameters $\beta$ for photoinduced energy transfer in different donor-bridge-acceptor molecules ${ }^{[8-10]}$

computational study, Eng and Albinsson find a good correlation between the distance dependence ( $\beta$-values) and donor-OPE energy differences $(\Delta \varepsilon)$ as predicted by Eqn. (2). Such electronic coupling effects caused by differences in donor-bridge energy gaps may in fact also account for the experimentally observed differences in distance dependences of energy transfer across OPE bridges: While Harriman et al. report $\beta=$ $0.11 \AA^{-1}$ for energy transfer through OPE connectors from a ruthenium(II) terpyridine complex to an osmium(II) terpyridine acceptor, ${ }^{99]}$ Eng et al. find $\beta=0.45 \AA^{-1}$ for energy transfer across the same bridges but between zinc and free base porphyrin donors and acceptors (Fig. 3). ${ }^{[10]}$

Additional experimental evidence for the importance of tunneling energy effects is provided by recent investigations of longdistance ET between randomly dispersed donors and acceptors in frozen glasses. ${ }^{[11,12]}$ In these studies, Gray, Winkler and coworkers investigated photoinduced electron tunneling from an excited iridium(I) complex to a benzoquinone acceptor; the glassing solvent served the purpose of the intervening medium, i.e. as a bridge for the long-range ET events. From time-resolved luminescence experiments it was possible to determine effective tunneling barriers for ET through different organic molecules (solvents). One of the key outcomes from this work is that the barrier for ET through toluene is $1.4 \mathrm{eV}$ whereas for 2-methyltetrahydrofuran it is $2.6 \mathrm{eV}$. Thus, the aromatic molecule with its relatively low lying $\pi^{*}$ levels mediates longrange electron tunneling significantly better than the saturated ether. This experimental result is in line with the theoretical predictions of the superexchange model for longdistance ET (Eqn. (2)).

In summary, three important conclusions regarding tunneling energy effects emerge from the studies cited in this contribution:

i) Almost 20 years after the landmark paper by Closs and Miller on the so-called inverted driving force effect, ${ }^{[2]}$ there now exists direct experimental evidence for the importance of tunneling energy effects in long-range ET. ${ }^{[6,7,11,12]}$

ii) The electronic D-A coupling for charge or energy transfer in molecular D-b-A systems is a function of the donor-bridge (or tunneling energy) gap already in the pre-resonance regime: Even long before the donor and bridge levels come into resonance and an incoherent hopping mechanism takes over, ${ }^{[5,13]}$ there is a strong dependence of superexchange ET rates on the D-b energy gap $\Delta \varepsilon ;{ }^{[8]}$

iii) Due to this fact and due to the relationship between $\beta$ and $\Delta \varepsilon$ (Eqn. (2)), , ${ }^{[4]}$ the distance decay parameter should be regarded as a system-specific (and not bridge-specific) parameter.

These findings may have important practical implications for obtaining longlived charge-separated states and are therefore directly relevant to solar energy conversion: The tunneling energy gaps for light-induced charge-separation $\left(\Delta \varepsilon_{\mathrm{CS}}\right)$ and thermal charge-recombination $\left(\Delta \varepsilon_{\mathrm{CR}}\right)$ need not be identical even in D-b-A molecules where the bridge is symmetrical. Notably, in a scenario where $\Delta \varepsilon_{\mathrm{CS}}<\Delta \varepsilon_{\mathrm{CR}}$ the electronic coupling $\mathrm{H}_{\mathrm{DA}}$ will be stronger for the energy-storing forward-ET than for the energy-wasting back-ET. In other words, tunneling energy effects may be exploited eventually to discriminate between the rates of desired and undesired charge transfers in artificial systems. The synthesis, characterization, and spectroscopic investigation of molecular model systems that display such properties represents a formidable challenge to experimental chemists. Our newly established research group at the University of Geneva has taken on this challenge.

\section{Acknowledgements}

Financial support from the Swiss National Science Foundation and from the Department of Inorganic, Analytical and Applied Chemistry at the University of Geneva is gratefully acknowledged. The author thanks Alan F. Williams for useful comments.

Received: October 18, 2007

[1] R. A. Marcus, N. Sutin, Biochim. Biophys. Acta 1985, 811, 265.

[2] G. L. Closs, J. R. Miller, Science 1988, 240, 440.

[3] L. S. Fox, M. Kozik, J. R. Winkler, H. B. Gray, Science 1990, 247, 1069.

[4] H. McConnell, J. Chem. Phys. 1961, 35, 508.

[5] W. B. Davis, W. A. Svec, M. A. Ratner, M R. Wasielewski, Nature 1998, 396, 60.

[6] F. D. Lewis, J. Q. Liu, W. Weigel, W. Rettig, I. V. Kurnikov, D. N. Beratan, Proc. Natl. Acad. Sci. USA 2002, 99, 12536.

[7] G. S. M. Tong, I. V. Kurnikov, D. N. Beratan, J. Phys. Chem. B 2002, 106, 2381.

[8] M. P. Eng, B. Albinsson, Angew. Chem., Int. Ed. 2006, 45, 5626.

[9] A. Harriman, A. Khatyr, R. Ziessel, A. C. Benniston, Angew. Chem., Int. Ed. 2000, 39, 4287.

[10] M. P. Eng, T. Ljungdahl, J. Mårtensson, B. Albinsson, J. Phys. Chem. B 2006, 110, 6483.

[11] O. S. Wenger, B. S. Leigh, R. M. Villahermosa, H. B. Gray, J. R. Winkler, Science 2005, 307, 99.

[12] O. S. Wenger, H. B. Gray, J. R. Winkler, Chimia 2005, 59, 94.

[13] R. H. Goldsmith, L. E. Sinks, R. F. Kelley, L. J. Betzen, W. H. Liu, E. A. Weiss, M. A. Ratner, M. R. Wasielewski, Proc. Natl. Acad. Sci. USA 2005, 102, 3540. 